\title{
Enhancement pattern of differentiated and undifferentiated gastric carcinoma on multiphasic contrast-enhanced computed tomography
}

\author{
Baphiralyne Wankhar ${ }^{1, A, B, C, D, E, F,}$, Aman Yusuf Khan ${ }^{1, A, B, C, D, E, F}$, Vandana Raphael ${ }^{2, A, B, D, E}$, Dathiadiam Tongper, $3, A, B, D, E$, \\ Donkupar Khongwar ${ }^{3, A, B, D, E}$, Kyrshanlang G Lynrah ${ }^{4, A, B, D, E}$
}

'Department of Radiology, Northeastern Indira Gandhi Regional Institute of Health and Medical Sciences, Shillong, Meghalaya, India ${ }^{2}$ Department of Pathology, Northeastern Indira Gandhi Regional Institute of Health and Medical Sciences, Shillong, Meghalaya, India ${ }^{3}$ Department of Surgery, Northeastern Indira Gandhi Regional Institute of Health and Medical Sciences, Shillong, Meghalaya, India ${ }^{4}$ Department of General Medicine, Northeastern Indira Gandhi Regional Institute of Health and Medical Sciences, Shillong, Meghalaya, India

\section{Abstract}

Purpose: To study the enhancement pattern of differentiated and undifferentiated gastric carcinoma on multiphasic contrast-enhanced computed tomography (CT).

Material and methods: Seventy patients with biopsy-proven gastric cancer underwent multiphasic contrast-enhanced CT. The CT protocol include plain, arterial, portal venous, and hepatic venous phase. Tumour size, location, peak-enhancement characteristics, and staging were evaluated.

Results: The peak-enhancement type was 'arterial' in 20 out of 28 within the differentiated-type GCAs and 'portalvenous' in 37 out of 42 within the undifferentiated-type GCAs ( $\chi^{2}$ statistic with Yates correction $=23.3981, p<0.00001$ ). The maximum attenuation value was statistically significant for the arterial phase between differentiated and undifferentiated GCAs $(p<0.05)$.

Conclusions: Assessing peak-enhancement in a multiphasic CT can help identify the histological subcategory of gastric carcinomas that has prognostic significance. Arterial phase peak-enhancement is frequently seen in differentiated carcinomas whereas venous phase peak-enhancement is seen in undifferentiated carcinomas.

Key words: gastric carcinoma, multiphasic CT, enhancement pattern.

\section{Introduction}

Gastric adenocarcinoma (GCA) is a common malignancy with a global prevalence. Lauren et al. classified GCAs into 3 broad categories on histopathology: intestinal (welldifferentiated), diffuse (poorly-differentiated), and indeterminate/mixed types [1]. Tumours in these categories exhibit differences in aetiology, genetics, and epidemiology $[2,3]$. Histological characterization is also one of the important prognostic factor in GCA [4]. The diffuse type is associated with an aggressive clinical course. Adachi et al. showed that the overall 5-year survival rate for patients with well-differentiated GCA was higher than that for patients with poorly-differentiated type (76\% vs. $67 \%$; $p=0.058)$, especially in patients with tumours $\geq 10 \mathrm{~cm}$ ( $42 \%$ vs. $14 \% ; p=0.017$ ) [4]. Currently, histological characterization of GCA is possible only by endoscopic-guided biopsy. However, endoscopy is an invasive procedure and has its limitations. The obtained biopsy may not be representative of the entire tumour, which is known to show geographical histological variations [5]. Furthermore, the extramural extent of the tumour, and distant

Correspondence address:

Kyrshanlang Giri Lynrah, Northeastern Indira Gandhi Regional Institute of Health and Medical Sciences, Shillong, Meghalaya, India, phone: 9436333266 , e-mail: rilawa23@rediffmail.com

Authors' contribution:

A Study design · B Data collection · C Statistical analysis · D Data interpretation · E Manuscript preparation · F Literature search · G Funds collection 
nodal and visceral metastases cannot be assessed by endoscopy.

Previous studies have attempted to differentiate histological types of GCA using computed tomography (CT) [6-11]. Tsurumaru et al. studied peak enhancement in GCAs using multiphasic contrast-enhanced CT gastrography (CE-CTG) [10]. They concluded that the 3 broad histological categories of GCA can be predicted by measuring the peak enhancement on a multiphasic CT. The purpose of our study was to examine the utility of multiphasic contrast-enhanced CT in differentiating the 3 broad histological categories of GCAs.

\section{Material and methods}

This was a prospective study approved by our institutional review board, and informed written consent was taken from all patients. Inclusion criteria included consecutive patients who underwent endoscopic-guided biopsy and showed histological evidence of GCA. Upper gastrointestinal endoscopy was performed in all 70 patients using an Olympus GIF-Q150 Actera. All 70 patients underwent subsequent multiphasic $\mathrm{CT}$ as part of clinical staging.

\section{Histopathological classification}

The tumours were graded into the following categories for our study: a) differentiated type, b) undifferentiated type, and c) mixed types. The following criteria were applied for the above classification. Differentiated type included well and moderately differentiated GCAs, and papillary adenocarcinomas. Undifferentiated type included poorly differentiated adenocarcinomas, signet-ring cell carcinomas, and mucinous variant. Mixed type were those with features of both differentiated and undifferentiated GCA.

\section{Multiphasic computed tomography protocol}

All 70 patients underwent multiphasic CT on a 128-slice multidetector scanner: SOMATOM Definition AS+, Siemens, Erlangen, Germany. Patient preparation included overnight fasting. To achieve adequate gastric distension, each patient was given $600-1000 \mathrm{ml}$ of water as negative contrast immediately before the exam in the CT suite. We did not administer any anti-peristaltic agent to our patients. Precontrast axial images of the upper abdomen were acquired using the following parameters: slice thickness and intervals $=5 \mathrm{~mm}$, rotation time $=0.5 \mathrm{~s}$, pitch $=1.5, \mathrm{kVp}=100$, $\mathrm{mAs}=170$, and matrix $=512 \times 512$. For triphasic post-contrast CT, $70-80 \mathrm{ml}$ of nonionic contrast medium (Iopromide $370 \mathrm{mg} / \mathrm{ml}$; Bayer AG, Germany) was administered intravenously using a power injector at the flow rate of 3.5-4 ml/s. Axial images of the upper abdomen were acquired during the arterial phase, the whole abdomen for portal-venous phase, and again the upper abdomen during the hepaticvenous phase using a $\mathrm{kVp}=120, \mathrm{mAs}=210$. The other ac- quisition parameters were similar to the pre-contrast scan. At our institution, the abdominal triphasic CT images are obtained at $21 \mathrm{~s}$ for arterial dominant phase, $32 \mathrm{~s}$ for portalvenous phase, and $50 \mathrm{~s}$ for hepatic-venous phase after the start of intravenous injection. The same timings were also applied for this study. We acquired axial images of the chest during the arterial phase to evaluate for lung metastases, but these images were not included for analysis. All axial acquisitions were reconstructed to $1 \mathrm{~mm}$ axial slice thickness. Coronal and sagittal reformations were reconstructed from the $1 \mathrm{~mm}$ axial reconstructions for all 3 phases.

\section{Image interpretation}

Two experienced radiologists in abdominal radiology reviewed the CT dataset. Both the radiologists were blinded to the histopathological findings but used endoscopic findings for reference. The following observations were recorded by the readers, and any disagreement was resolved by consensus.

Size of gastric lesion: The largest 2 orthogonal dimensions were recorded for each lesion by the readers and were averaged.

Tumour location: For the purpose of analysis, the stomach was anatomically divided into 3 portions: upper $(\mathrm{U})$, middle $(\mathrm{M})$, and lower $(\mathrm{L})$ segments, by the lines connecting the trisected points on the lesser and greater curvatures [12]. Gastric tumours were classified by the segments involved as $\mathrm{U}, \mathrm{M}$, or L. If more than 1 segment was involved, the tumours were classified by the segment containing the bulk of the tumour. If all 3 segments were involved, the tumour was classified as diffuse (D). Oesophageal and duodenal extension of the tumours were also recorded as ES and DU, respectively.

Tumour peak-enhancement characteristics: The readers manually traced regions of interest (ROIs) at the maximum attenuation value (HU) for the pre-contrast and all 3 postcontrast phase images. The maximum values (HU) were averaged for the readers. The peak-enhancement phase for the tumours were classified as 'arterial', 'portal-venous', and 'hepatic-venous' based on the phase with the maximum attenuation value. The chi-square test was performed to determine the association of enhancement pattern between differentiated and undifferentiated tumours. Independent samples T-test was applied to determine the predictability of contrast study for differentiating the type of GCA.

Tumour, Node, and Metastasis staging: $8^{\text {th }}$ American Joint Committee on Cancer (AJCC) staging was followed for staging purposes [13].

\section{Results}

The study included 70 patients who were positive for GCA after endoscopic-guided biopsy. The 70 patients included 47 males. Mean age was 52.5 years $(S D \pm 13.3)$. The tumour location, histopathology, and clinical TNM staging for the 
70 patients are summarized in Table 1 . Notably, there was no mixed type on histopathological analysis in our cohort. The tumour maximum dimensions varied between $2.7 \mathrm{~cm}$ and $12.0 \mathrm{~cm}$. The majority of the tumours were predominantly located in the lower gastric segment in both the differentiated and undifferentiated types ( $n=25 / 28$ for differentiated GCAs and $n=27 / 42$ for undifferentiated GCAs). Diffuse GCAs involving all 3 segments were seen only in the undifferentiated type $(n=3 / 42)$. All 70 GCAs in our series were staged $\geq$ IIB.

\section{Peak-enhancement characteristic}

The peak-enhancement type was 'arterial' in 20 out of 28 within the differentiated-type GCAs and 'portal-venous' in 37 out of 42 within the undifferentiated-type GCAs $\left(\chi^{2}\right.$ statistic with Yates's correction $\left.=23.3981, p<0.00001\right)$ (Table 2 ). The mean of the maximum attenuation values (HU) for 70 GCA on multiphasic CT is summarized in Table 3. Mean maximum attenuation value (HU) was higher in the arterial phase for differentiated GCAs and in the portal-venous phase for undifferentiated GCAs.
The maximum attenuation value was statistically significant for the arterial phase between differentiated and undifferentiated GCAs $(p \leq 0.05)$. There was no statistical significance for portal-venous $(p=0.08)$ and hepatic-venous $(p \leq 0.19)$ phases (Supplemental Table).

There was no significant statistical difference in lymph nodal and hepatic metastasis between the differentiated and undifferentiated carcinomas in our study $\left(\chi^{2}\right.$ statistic with Yates's correction $=1.7414, p=0.186$ for lymph node metastasis; $\chi^{2}$ statistic with Yates's correction $=0.0076$, $p=0.930$ for hepatic metastasis).

\section{Discussion}

Adachi et al. studied the microvascular architecture of GCA in relation to histologic subtypes. They observed that on histopathology, the differentiated carcinomas were frequently normovascular (65\%) or hypervascular (24\%), whereas undifferentiated carcinomas were hypovascular (60\%). They believed that stromal hypervascularity in welldifferentiated GCA may partly account for its propensity to metastasize to liver and lung [14]. However, in our study,

Table 1. Histopathological type, location, extent, and tumour staging for $N=70$ with gastric adenocarcinoma

\begin{tabular}{|c|c|c|c|c|c|c|}
\hline \multirow{2}{*}{$\begin{array}{l}\text { Histological } \\
\text { subtype } \\
(N=70)\end{array}$} & \multirow{2}{*}{$\begin{array}{l}\text { Predominant gastric } \\
\text { segment location } \\
\text { of the tumour }\end{array}$} & \multirow{2}{*}{$\begin{array}{c}\text { No. of patients } \\
\text { with oesophageal (ES)/ } \\
\text { duodenal (DU) involvement }\end{array}$} & \multirow{2}{*}{$\begin{array}{l}\text { No. of patients } \\
\text { with lymph nodal } \\
\text { involvement }\end{array}$} & \multirow{2}{*}{$\begin{array}{l}\text { No. of patients } \\
\text { with hepatic } \\
\text { metastasis }\end{array}$} & \multicolumn{2}{|c|}{ Tumour staging } \\
\hline & & & & & TNM stage & $n$ \\
\hline \multirow{5}{*}{$\begin{array}{l}\text { Differentiated type } \\
(n=28)\end{array}$} & Upper $=0$ & $\mathrm{ES}=$ nil & $10 / 28$ & $2 / 28$ & $\leq \| \mathrm{A}$ & 0 \\
\hline & Middle $=3$ & \multirow[t]{4}{*}{$\mathrm{DU}=2$} & & & $\| B$ & 11 \\
\hline & Lower $=25$ & & & & III & 5 \\
\hline & \multirow[t]{2}{*}{ Diffuse $=0$} & & & & IVA & 8 \\
\hline & & & & & IVB & 4 \\
\hline \multirow{5}{*}{$\begin{array}{l}\text { Undifferentiated type } \\
(n=42)\end{array}$} & Upper = 2 & $\mathrm{ES}=2$ & $23 / 42$ & $4 / 42$ & $\leq \| \mathrm{A}$ & 0 \\
\hline & Middle $=10$ & \multirow[t]{4}{*}{$\mathrm{DU}=6$} & & & $\| B$ & 11 \\
\hline & Lower $=27$ & & & & III & 7 \\
\hline & \multirow[t]{2}{*}{ Diffuse $=3$} & & & & IVA & 11 \\
\hline & & & & & IVB & 13 \\
\hline
\end{tabular}

Table 2. Peak-enhancement pattern on multiphasic computed tomography for $N=70$ gastric adenocarcinomas

\begin{tabular}{|l|c|c|}
\hline \multirow{2}{*}{ Histological type } & \multicolumn{2}{|c|}{ Peak-enhancement type } \\
\cline { 2 - 3 } & Arterial & Portal-venous \\
\hline Differentiated type, $n=28$ & 20 & 37 \\
\hline Undifferentiated type, $n=42$ & 5 & 37 \\
\hline
\end{tabular}

Table 3. Maximum attenuation value (HU) on multiphasic $\mathrm{CT}$ for $70 \mathrm{GCAs}$

\begin{tabular}{|l|c|c|c|c|}
\hline Histopathological type & $\begin{array}{c}\text { Pre-contrast, } \\
\text { mean }( \pm \text { SD) }\end{array}$ & $\begin{array}{c}\text { Arterial phase, } \\
\text { mean }( \pm \text { SD })\end{array}$ & $\begin{array}{c}\text { Portal-venous phase, } \\
\text { mean }( \pm \text { SD })\end{array}$ & $\begin{array}{c}\text { Hepatic-venous phase, } \\
\text { mean }( \pm \text { SD })\end{array}$ \\
\hline Undifferentiated $(n=42)$ & $37.31( \pm 5.74)$ & $55.10( \pm 10.89)$ & $69.00( \pm 12.18)$ & $47.31( \pm 10.98)$ \\
\hline Differentiated $(n=28)$ & $38.14( \pm 5.69)$ & $70.36( \pm 16.72)$ & $64.04( \pm 10.61)$ & $50.89( \pm 11.45)$ \\
\hline Total $(N=70)$ & $37.64( \pm 5.70)$ & $61.20( \pm 15.38)$ & $67.01( \pm 11.76)$ & $48.74( \pm 11.23)$ \\
\hline
\end{tabular}


we did not observe a statistically significant difference in hepatic metastasis between differentiated and undifferentiated GCAs (Table 1) (Yates's correlation $=0.0076, p=0.930$ ).

The enhancement characteristics of GCAs have been a subject of interest in previous studies with implications on tumour detection and staging, and with reported differences among the histological categories[6-11,15-18]. Undifferentiated GCAs carry a poor prognosis. Hence, the ability to predict histology based on CT characteristics may add clinical value. In our study, there was a statis-
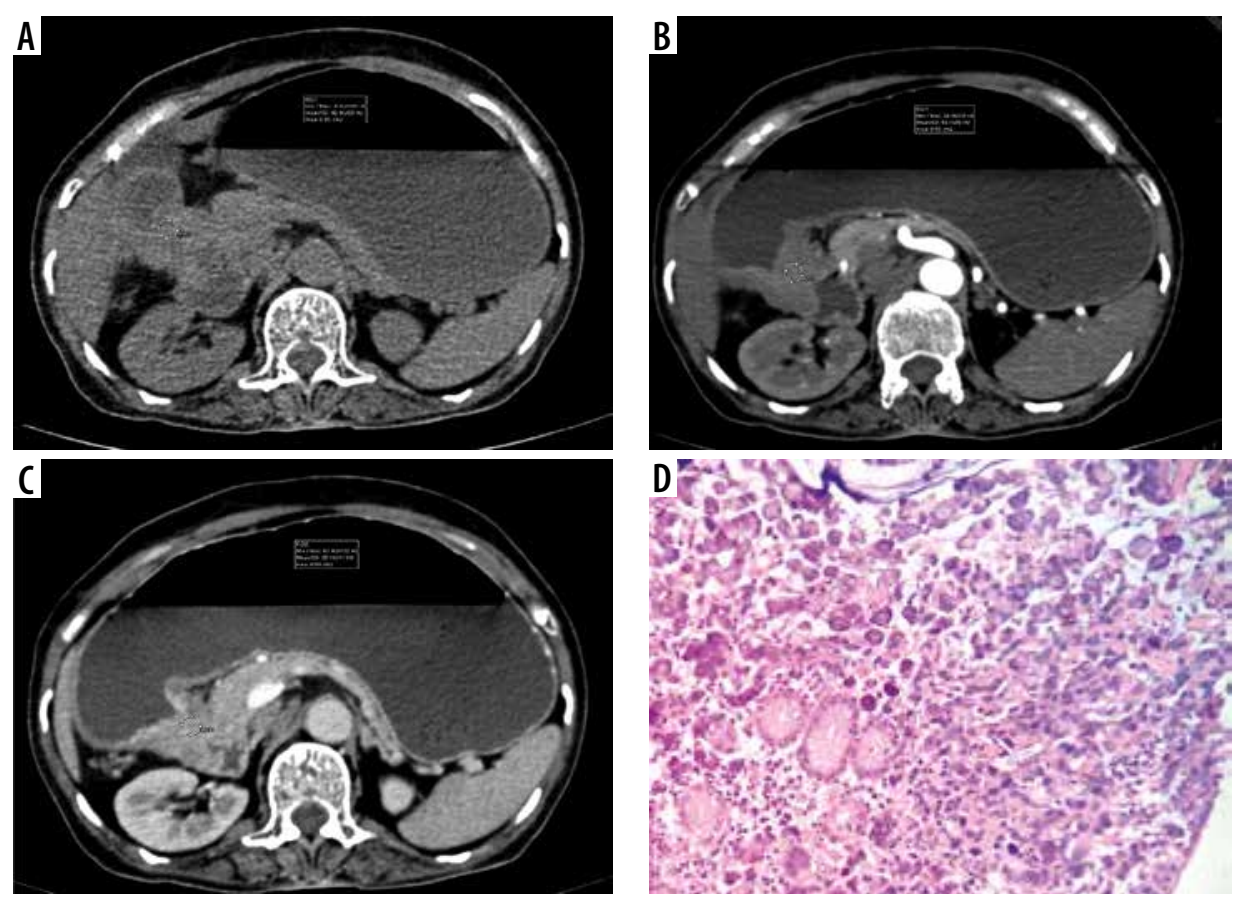

Figure 1. Typical undifferentiated: Undifferentiated-type gastric cancer in a 65-year-old woman. A-C) Contrast-enhanced computed tomography abdomen showed a $4.1 \times 2.7 \mathrm{~cm}$ mass in the antropyloric region. Regions of interest (ROI) are drawn in the area having the highest attenuation value. The computed tomography enhancement peaked in the portal phase, and the attenuation values were $58 \mathrm{HU}$ in the arterial phase and $85 \mathrm{HU}$ in the portal phase. D) Photomicrograph (original magnification $\times 400$ ) shows poorly differentiated adenocarcinoma
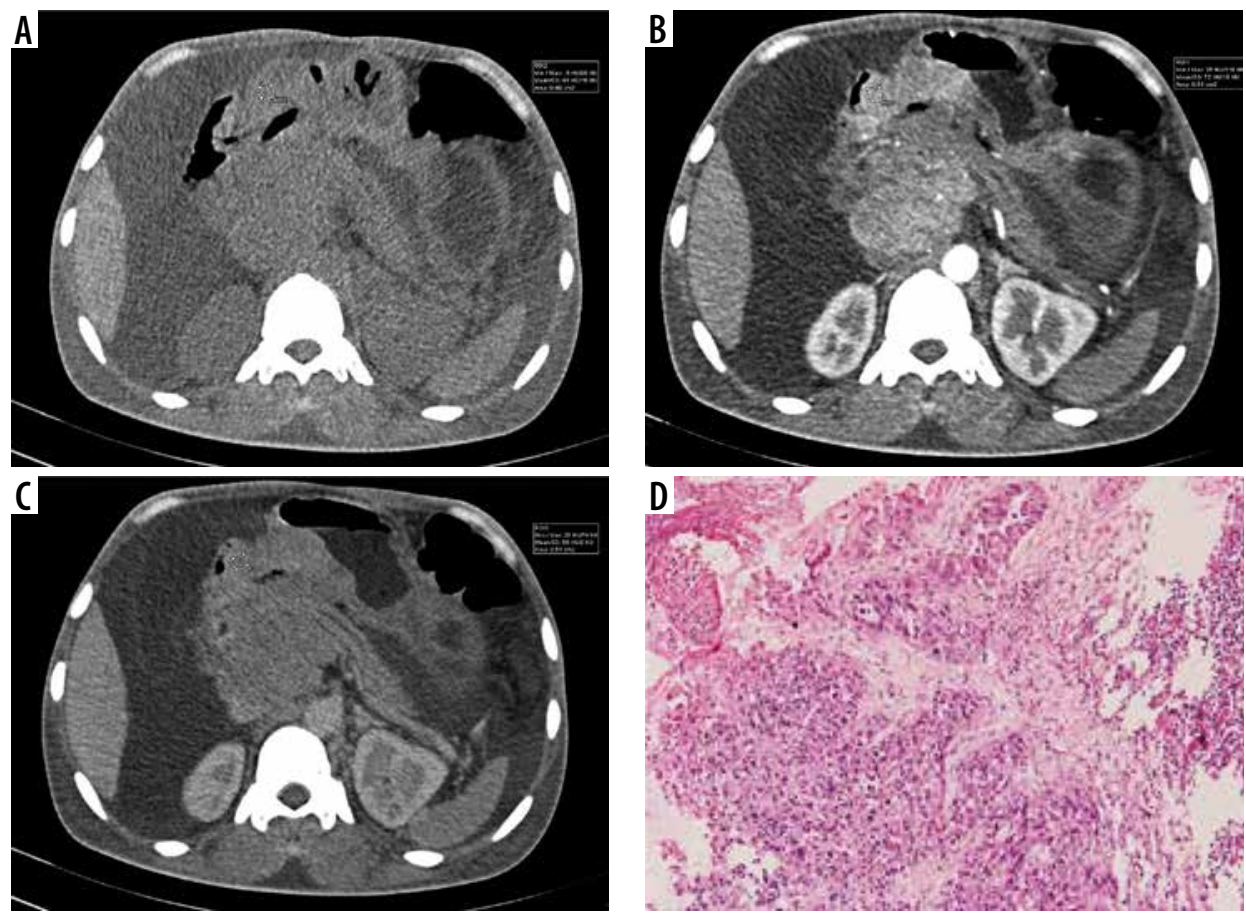

Figure 2. Typical differentiated: Differentiated-type gastric cancer in a 60-year-old man. A-C) Contrast-enhanced computed tomography abdomen showed a large $8.5 \times 3.7 \mathrm{~cm}$ enhancing mass in the antrum (circle ROI). The computed tomography enhancement peaked in the arterial phase and the attenuation values were $72 \mathrm{HU}$ in the arterial phase and $56 \mathrm{HU}$ in the portal phase. D) Photomicrograph (original magnification $\times 100$ ) shows differentiated adenocarcinoma 
tically significant difference in the enhancement pattern between differentiated and undifferentiated GCAs. An arterial phase dominant enhancement was present in $71.4 \%$ of the differentiated GCAs based on the maximum attenuation value measured within the tumour (Figures 2, 4, 5 and 8 ). A portal-phase dominant enhancement was observed in $88 \%$ of undifferentiated GCAs (Figures 1, 3, 6 and 7).Our observations again probably reflect the microvascular architecture of these tumours, as previously reported by Adachi et al. [14].
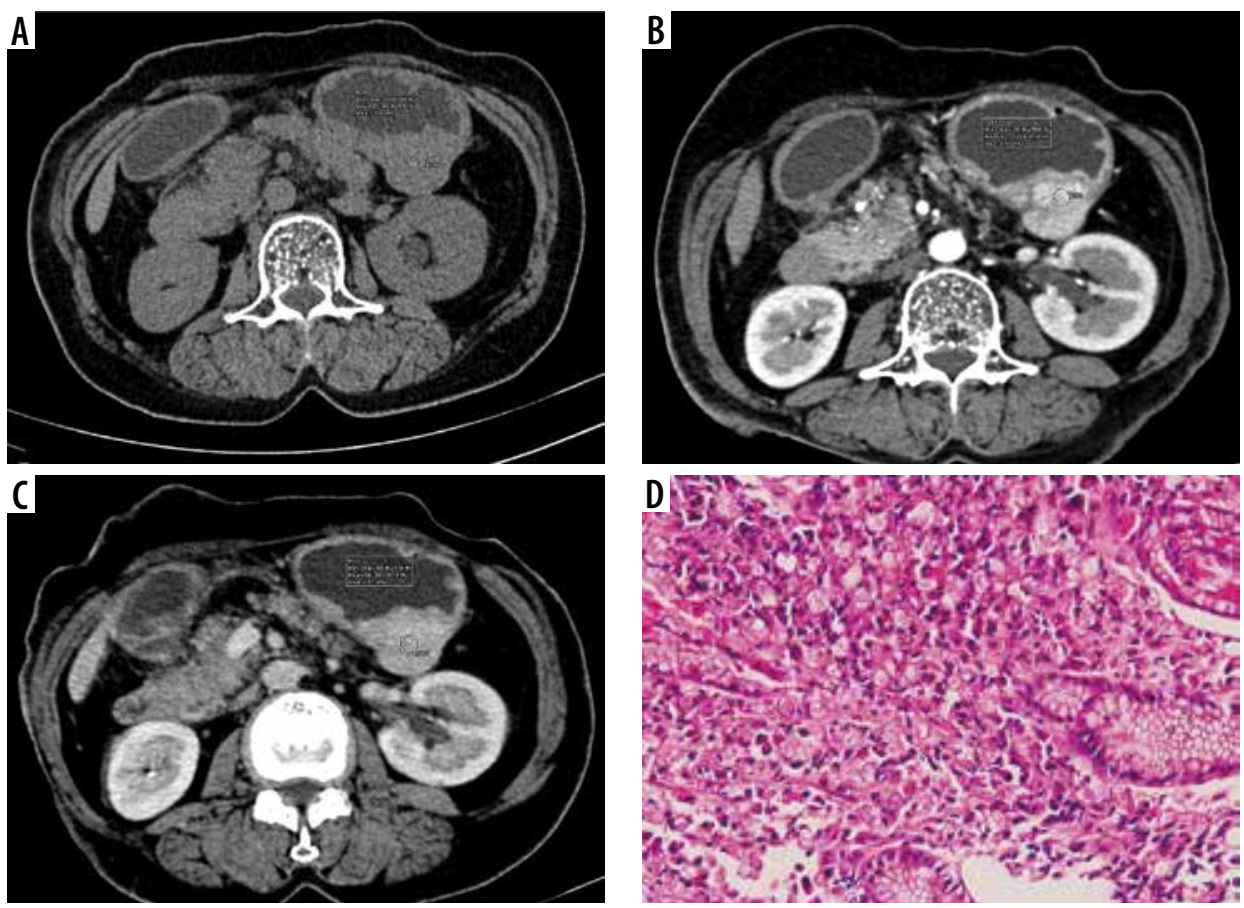

Figure 3. Atypical undifferentiated: Atypical enhancement pattern of undifferentiated-type gastric cancer in a 65-year-old woman. A-C) (ontrast-enhanced computed tomography abdomen showed focal thickening measuring $5 \times 4.6 \mathrm{~cm}$ in the posterior wall of the stomach (circle ROl). The computed tomography enhancement peaked in the arterial phase, and the attenuation values were $122 \mathrm{HU}$ in the arterial phase, $88 \mathrm{HU}$ in the portal phase. D) Photomicrograph (original magnification $\times 200$ ) shows poorly differentiated adenocarcinoma with signet ring cells
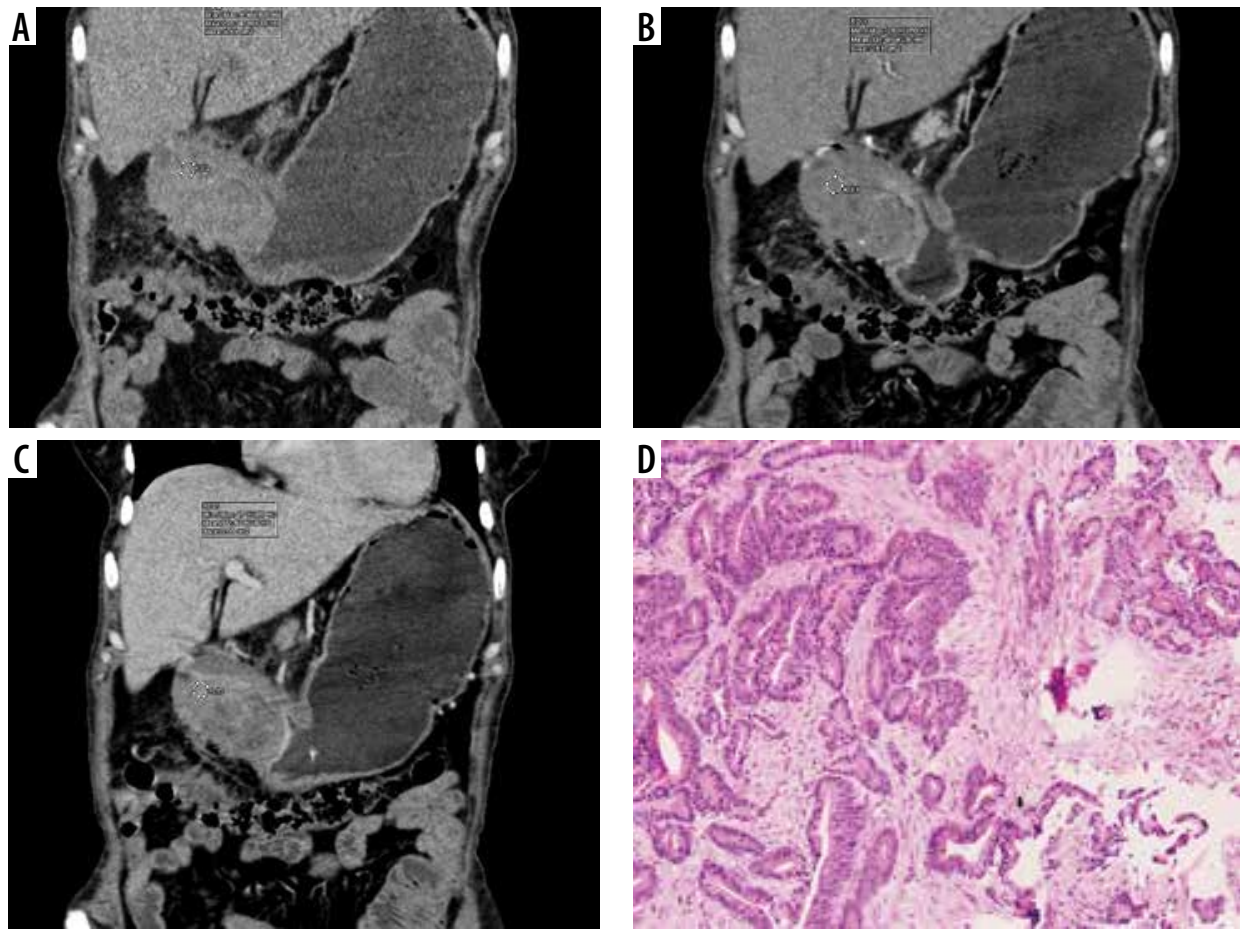

Figure 4. Atypical differentiated: Atypical enhancement pattern of differentiated-type gastric cancer in a 67-year-old woman. A-C) (ontrast-enhanced computed tomography abdomen coronal MPR image showed a $6 \times 3.8 \mathrm{~cm}$ mass in the antrum (circle ROI). The computed tomography enhancement peaked in the portal phase and the attenuation values were $47 \mathrm{HU}$ in the arterial phase and $63 \mathrm{HU}$ in the portal phase. D) Photomicrograph (original magnification $\times 100)$ shows differentiated adenocarcinoma 

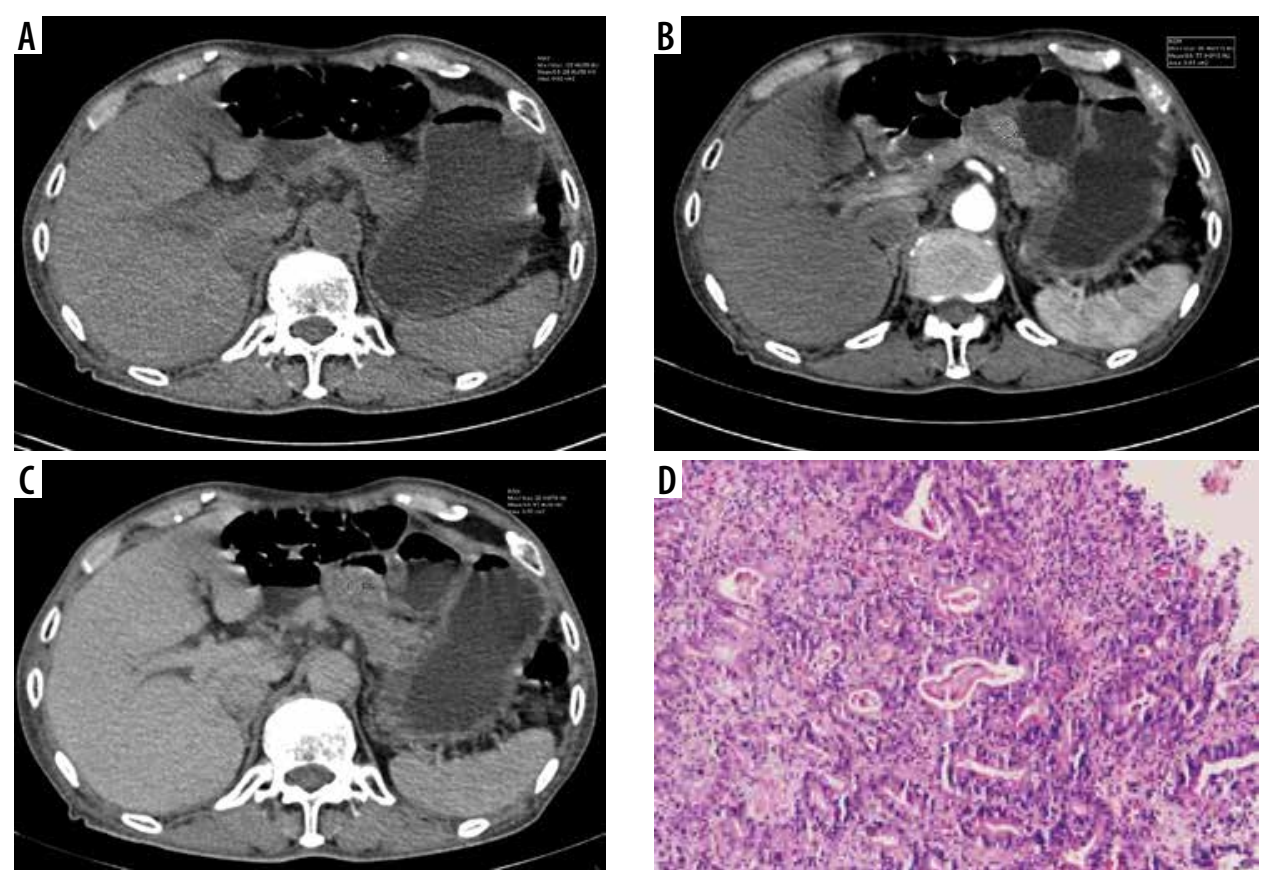

Figure 5. Typical differentiated: Differentiated-type gastric cancer in a 89-year-old man. A-C) Contrast-enhanced computed tomography abdomen showed focal thickening in the posterior wall of stomach (circle ROI). The computed tomography enhancement peaked in the arterial phase, and the attenuation values were $77 \mathrm{HU}$ in the arterial phase and $57 \mathrm{HU}$ in the portal phase. D) Photomicrograph (original magnification $\times 100$ ) shows differentiated adenocarcinoma
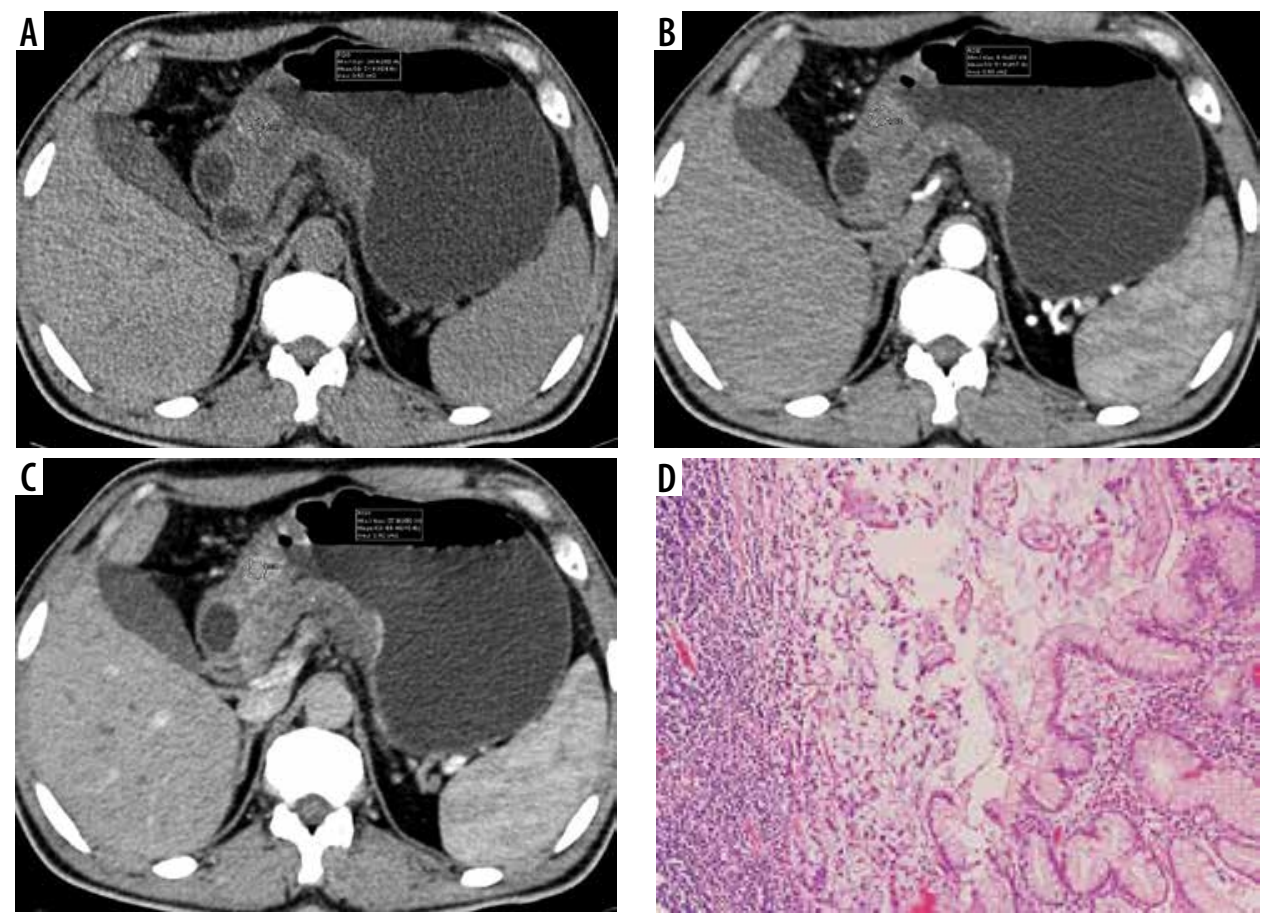

Figure 6. Typical undifferentiated: Undifferentiated type in a 43-year-old man. A-C) Contrast-enhanced computed tomography abdomen showed circumferential thickening in the antropyloric region (circle ROI) measuring $8.4 \times 3.3 \mathrm{~cm}$. The computed tomography enhancement peaked in the portal phase, and the attenuation values were $51 \mathrm{HU}$ in the arterial phase and $68 \mathrm{HU}$ in the portal phase. D) Photomicrograph (original magnification $\times 200$ ) shows poorly differentiated adenocarcinoma-mucinous type

In a previous similar study, Tsurumaru et al. retrospectively examined 47 patients with advanced GCAs (T2-T4) using multiphasic $\mathrm{CT}$ with arterial, portal venous, and delayed-phase images at $40 \mathrm{~s}, 70 \mathrm{~s}$, and $240 \mathrm{~s}$, respectively. They reported statistically significant higher CT attenua- tion values in undifferentiated GCAs in the delayed phase compared to mixed and differentiated types $(p=0.004)$. Using a cut-off of $109.8 \mathrm{HU}$, the maximum accuracy for undifferentiated GCAs in the delayed phase in their study was $72.3 \%$ [10]. Contrary to our observations, they did not 

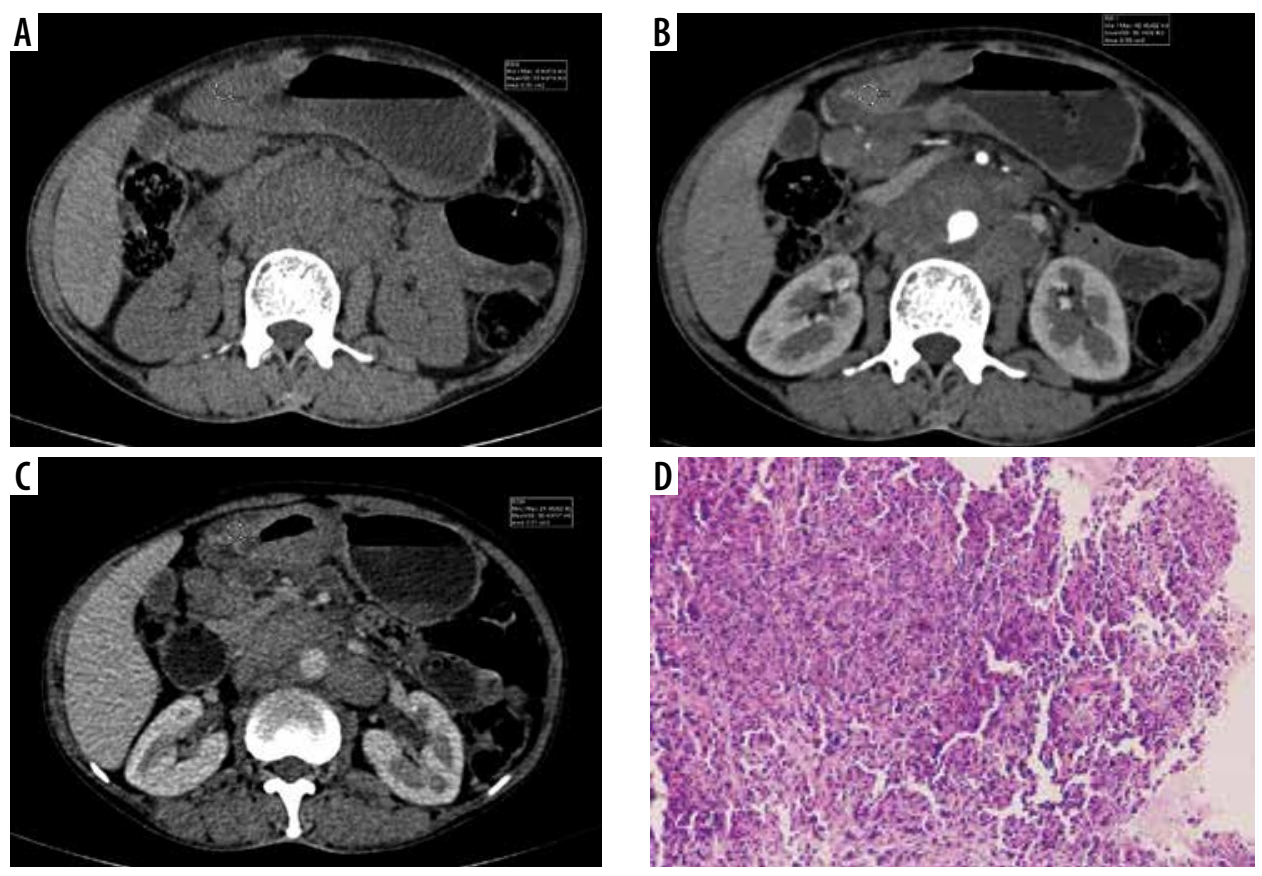

Figure 7. Atypical undifferentiated: Atypical enhancement pattern of undifferentiated-type gastric cancer in a 35-year-old woman. A-C) Contrast-enhanced computed tomography abdomen showed circumferential thickening in the antrum (circle ROI) measuring $6.7 \times 2.4 \mathrm{~cm}$ in size. The computed tomography enhancement peaked in the arterial phase, and the attenuation values were $66 \mathrm{HU}$ in the arterial phase and $56 \mathrm{HU}$ in the portal phase. D) Photomicrograph (original magnification $\times 100$ ) shows poorly differentiated adenocarcinoma
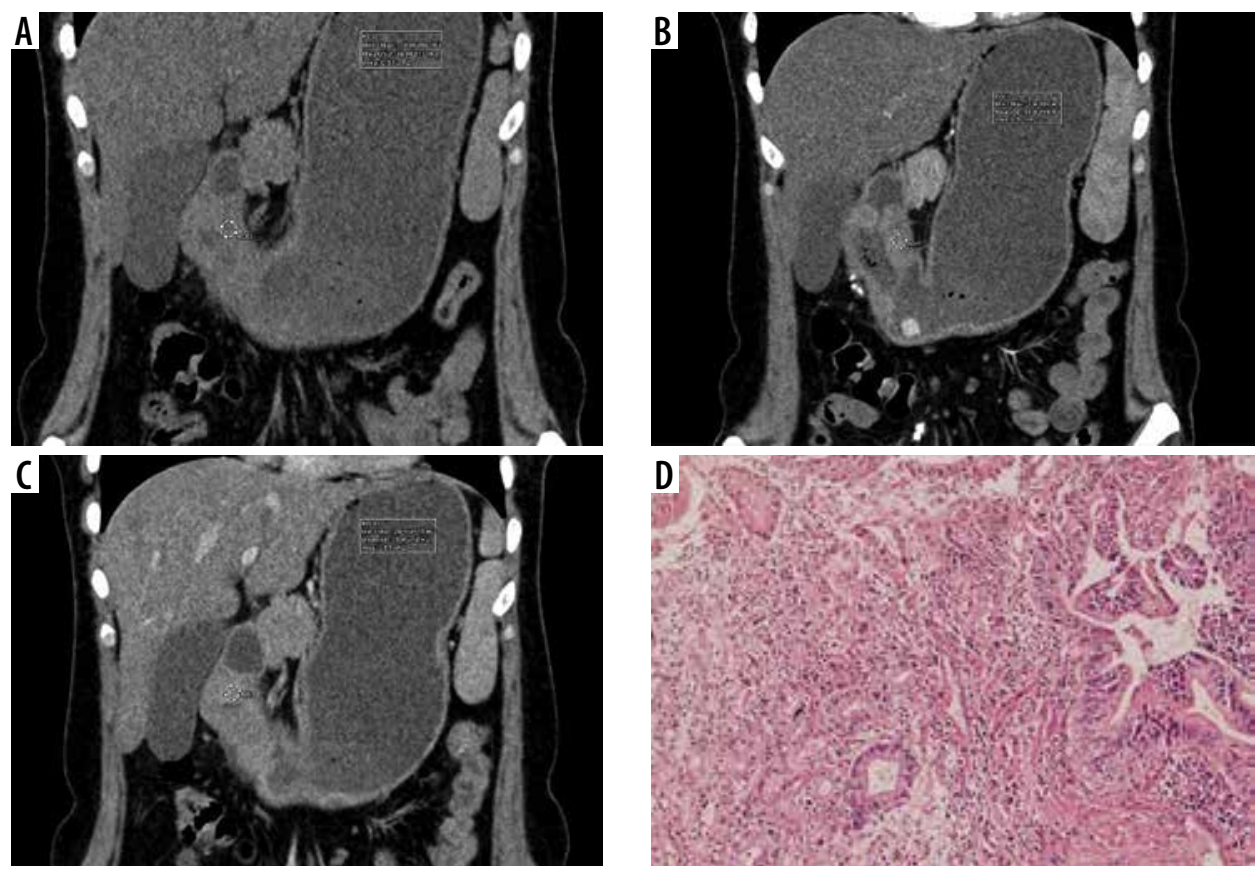

Figure 8. Atypical differentiated: Atypical enhancement pattern of differentiated-type gastric cancer in a 40-year-old woman. A-C) Contrast-enhanced computed tomography abdomen coronal MPR image showed a $8.1 \times 3.1 \mathrm{~cm}$ mass in the antropyloric region (circle ROI). The computed tomography enhancement peaked in the portal phase and the attenuation values were $55 \mathrm{HU}$ in the arterial phase and $76 \mathrm{HU}$ in the portal phase. D) Photomicrograph (original magnification $\times 100$ ) shows differentiated adenocarcinoma.

find significant difference in the arterial phase between the histological types. However, a direct comparison to their study may be limited due to differences in the CT protocol applied.

In a different study, Tsurumaru et al. retrospectively studied the enhancement pattern specifically in 21 diffuse- type advanced GCAs (histologically poorly differentiated adenocarcinomas) using dual-phasic CT [9]. They observed a double-layered pattern in all 21 cases in the arterial phase with a high attenuating inner layer. Such a pattern was not seen in our study. Also, there was no comparison with differentiated GCAs in their study and 
the mean attenuation value was employed for analysis rather than the maximum attenuation value.

Lee et al. studied the portal-venous phase enhancement characteristics in 81 gastric cancers ( 35 with signet ring cell carcinoma [SRC] and 45 non-signet ring cell carcinoma [NSRC]) [6]. They observed high-degree contrast enhancement (higher than liver) more frequently in SRC than in NSRC (37.1\% vs. $15.6 \%$, respectively) in the portalvenous phase. They attributed this to the presence of immature fibrosis in SRCs, which are considered a subset of diffuse GCAs. In our study, we did not observe a significant difference in enhancement pattern in the venous phase in the 8 cases of diffuse GCAs with signet ring histology compared to the rest.

Our study has limitations. First, this was a single-institution study with a relatively small sample size. Secondly, we only included the broad histological categories of GCA for our analysis and did not analyse the subtypes within these broad categories. Also, our cohort did not have mixed type for analysis. Thirdly, we only measured the maximum attenuation value within the tumour for analysis and did not consider the mean attenuation of the entire tumour. Given the known heterogeneity in tumours, this may not be a correct representation of the microvascular architecture. Finally, the definition of arterial and venous phases in our CT protocol is discordant with other previously reported studies, which makes direct comparison difficult.

\section{Conclusions}

Assessing peak enhancement in a multiphasic CT exam can help suggest the histological subcategory of gastric carcinomas, which has prognostic significance. Arterial phase peak-enhancement is frequently seen in differentiated carcinomas, whereas venous phase peak-enhancement in undifferentiated carcinomas. This is probably reflective of their microvascular architecture as reported in previous studies.

\section{Conflicts of interest}

The authors report no conflict of interest.

\section{References}

1. Lauren P. The two histological main types of gastric carcinoma: diffuse and so-called intestinal-type carcinoma. an attempt at a histo-clinical classification. Acta Pathol Microbiol Scand 1965; 64: 31-49.

2. Solcia E, Fiocca R, Luinetti $\mathrm{O}$, et al. Intestinal and diffuse gastric cancers arise in a different background of Helicobacter pylori gastritis through different gene involvement. Am J Surg Pathol 1996; 20 Suppl 1: S8-22.

3. Shah MA, Khanin R, Tang L, et al. Molecular classification of gastric cancer: a new paradigm. Clin Cancer Res Off J Am Assoc Cancer Res 2011; 17: 2693-2701.

4. Adachi Y, Yasuda K, Inomata M, et al. Pathology and prognosis of gastric carcinoma: well versus poorly differentiated type. Cancer 2000; 89: 1418-1424.

5. Hu B, El Hajj N, Sittler S, et al. Gastric cancer: classification, histology and application of molecular pathology. J Gastrointest Oncol 2012; 3: 251-261.

6. Lee JH, Park MS, Kim KW, et al. Advanced gastric carcinoma with signet ring cell carcinoma versus non-signet ring cell carcinoma: differentiation with multidetector CT. J Comput Assist Tomogr 2006; 30: $880-884$.

7. Takao M, Fukuda T, Iwanaga S, et al. Gastric cancer: evaluation of triphasic spiral CT and radiologic-pathologic correlation. J Comput Assist Tomogr 1998; 22: 288-294.

8. Tsurumaru D, Nishimuta Y, Muraki T, et al. Gastric cancer with synchronous and metachronous hepatic metastasis predicted by enhancement pattern on multiphasic contrast-enhanced CT. Eur J Radiol 2018; 108: 165-171.

9. Tsurumaru D, Miyasaka M, Muraki T, et al. Diffuse-type gastric cancer: specific enhancement pattern on multiphasic contrast-enhanced computed tomography. Jpn J Radiol 2017; 35: 289-295.
10. Tsurumaru D, Miyasaka M, Muraki T, et al. Histopathologic diversity of gastric cancers: Relationship between enhancement pattern on dynamic contrast-enhanced CT and histological type. Eur J Radiol 2017; 97: 90-95.

11. Tsurumaru D, Miyasaka M, Nishimuta Y, et al. Differentiation of early gastric cancer with ulceration and resectable advanced gastric cancer using multiphasic dynamic multidetector CT. Eur Radiol 2016; 26 : 1330-1337.

12. Japanese Gastric Cancer Association. Japanese classification of gastric carcinoma: 3rd English edition. Gastric Cancer 2011; 14: 101-112.

13. Amin MB (ed.). AJCC Cancer Staging Manual, Eighth Edition. American College of Surgeons; 2018.

14. Adachi Y, Mori M, Enjoji M, et al. Microvascular architecture of early gastric carcinoma. Microvascular-histopathologic correlates. Cancer 1993; 72: 32-36.

15. Lee JH, Jeong YK, Kim DH, et al. Two-phase helical CT for detection of early gastric carcinoma: importance of the mucosal phase for analysis of the abnormal mucosal layer. J Comput Assist Tomogr 2000; 24: 777-782.

16. Wei F, Huang P, Li S, et al. Enhancement patterns of gastric carcinoma on contrast-enhanced ultrasonography: relationship with clinicopathological features. PLoS One 2013; 8: e73050. DOI: 10.1371/ journal.pone.0073050.

17. Yu T, Wang X, Zhao Z, et al. Prediction of T stage in gastric carcinoma by enhanced CT and oral contrast-enhanced ultrasonography. World J Surg Oncol 2015; 13: 184.

18. Lee DH, Seo TS, Ko YT. Spiral CT of the gastric carcinoma: staging and enhancement pattern. Clin Imaging 2001; 25: 32-37. 